\title{
Artifact-free quantitative cardiovascular PET/MR imaging: An impossible dream?
}

\author{
Habib Zaidi, PhD, ${ }^{\mathrm{a}, \mathrm{b}, \mathrm{c}}$ and Rene Nkoulou, $\mathrm{MD}^{\mathrm{a}, \mathrm{d}}$ \\ a Division of Nuclear Médicine and Molecular Imaging, Geneva University Hospital, Geneva, \\ Switzerland \\ b Geneva University Neurocenter, University of Geneva, Geneva, Switzerland \\ c Department of Nuclear Medicine and Molecular Imaging, University of Groningen, Groningen, \\ Netherlands \\ d Division of Cardiology, Geneva University Hospital, Geneva, Switzerland
}

Received Nov 30, 2017; accepted Nov 30, 2017

doi: $10.1007 /$ s12350-017-1163-x

\section{See related article, 1107-1118}

Building a winning team requires a bit of complementarity and convincing common goals to overcome the frustration of being tied together. In the journey of cardiac PET/MRI, this frustration has a name: less optimal attenuation correction (AC) as compared to its PET/CT counterpart where CT-based AC works reasonably well. AC has been a key component in the development of nuclear cardiology providing at the same time more reliable assessment of regions with relative tracer abnormalities and the ground for accurate quantification of tracer uptake. ${ }^{1}$ It is therefore no wonder that reproducing the same level of confidence in $\mathrm{AC}$ using MRI as achieved using CT has been a major drive in the research over PET/MRI in general and in cardiovascular imaging applications, in particular.

MRI segmentation-based approaches have focused on deriving attenuation maps from basic MR sequences, such as T1-weighted or DIXON sequences; the latter consists of in- and opposed-phase images enabling segmentation in four tissue types (air, soft tissue, lung, and fat). ${ }^{2}$ Among the limitations of this approach, when using these sequences, is the inconsistent differentiation between air and bone densities. This is important when having in mind the position of the patient during thoracic PET/MRI with the arms laying aside the body to

Reprint requests: Rene Nkoulou, Division of Cardiology, Geneva University Hospital, Geneva, Switzerland; rene.nkoulou@hcuge.ch J Nucl Cardiol 2019;26:1119-21.

$1071-3581 / \$ 34.00$

Copyright (C) 2018 American Society of Nuclear Cardiology. promote optimal positioning of the radiofrequency surface coils and the relatively small field of view of MR images (only $45-50 \mathrm{~cm}$ compared to $60-70 \mathrm{~cm}$ on contemporary PET/CT systems) that systematically brings about truncation artifacts. A number of strategies enabling to incorporate bone density have been proposed and potential solutions, such as ultrashort echo time and zero echo time sequences, have been proposed and refined and are finding their way to the clinic in the context of brain imaging. ${ }^{3,4}$ In theory, incorporating an increased number of tissue types in the MRI-derived attenuation map would allow reducing quantification errors within the range of 5\% compared to CT-derived attenuation maps as proposed by Keereman et al using five tissue types. ${ }^{5}$

The recent advances in detector technology embedded in the latest designs of PET/MRI systems have contributed to the revival of an old concept referred to as joint estimation of activity and attenuation maps from emission data. This method has capitalized on time-of-flight capability to refine the positioning information on PET lines of responses for improved signalto-noise ratio. ${ }^{6}$ The attenuation maps could therefore be derived from the emission data using maximum likelihood reconstruction of activity and attenuation (MLAA) type of algorithms that are penalized using priors to reduce the cross-talk between activity and attenuation estimations. The scaling issue inherent in this approach has been recently dealt with using MLAA with a Gaussian mixture model approach. ${ }^{7}$ Noteworthily, this category of techniques is now routinely integrated by one of the manufacturers for the compensation of truncation artifacts.

The article of Lassen et al in this issue of the Journal of Nuclear Cardiology ${ }^{\circledR}$ discusses the frequency of AC- 
generated artifacts during cardiac PET/MRI in 20 patients referred for the assessment of rest perfusion and viability. ${ }^{8}$ Simultaneous PET and MRI acquisitions were performed on an mMR system with list mode data acquisition of rest ${ }^{13} \mathrm{~N}$-ammonia and ${ }^{18} \mathrm{~F}$-FDG preceded by MRI acquisition using a DIXON sequence. They used a commercial segmentation-based AC method using the standard DIXON sequence and routinely integrated MLAA-based truncation correction. A systematic review of the MRI-derived attenuation maps and attenuation-corrected PET data allowed the identification of the four main types of AC-related artifacts, namely misalignment (occurring in $80 \%$ of the cases), susceptibility $(50 \%)$, tissue inversion $(30 \%)$, and total lung volume change $(25 \%)$. Of particular importance was the fact that a substantial proportion of patients presented with multiple artifacts and that suitable correction could not be proposed for tissue inversion. These translated into almost $15 \%$ of patients having falsepositive defects that could eventually be corrected after additional rigid-body co-registration or retrospective filling of the susceptibility gaps.

A preliminary appraisal lies in the fact that, in this study, the acquisition protocol that is representative of conventional cardiac PET/MRI examinations promoted $80 \%$ of misalignment. Misalignment is not a specificity of PET/MRI since this may also occur during cardiac PET/CT in as many as $40 \%$ of cases, thus impacting the clinical outcome in almost $20 \%$ of cases. ${ }^{9,10}$ Respiratory misregistration is only an aspect of this and partial response would follow from more systematic implementation of recent advances in respiratory and cardiac motion compensation algorithms during PET/MR acquisitions. ${ }^{11,12}$ Yet, the most effective way to reduce misalignment lies in comfortable positioning of the patient and refraining from overextended acquisition protocols. This is appealing in PET/MRI to maximize the information collected and in part related to a pressure to provide more comprehensive results to justify the investment in PET/MRI clinical and research programs.

Another lesson learned would be that in cardiac PET/MRI, artifacts hardly come isolated. Therefore, despite their easy recognition and the availability of numerous compensation methods that can be used to correct them, multiple corrections eventually introduce inhomogeneity, thus hampering optimal quantification. That is particularly true if we keep in mind that opposite to the simplistic segmentation of the myocardium as a single tissue type with homogenous density, scarred myocardium would display different tissue density as compared to viable myocardium and incorporation of this information similar to when using PET/CT yields more accurate assessment of the metabolic information. ${ }^{13,14}$ Caution shall still prevail as more systematic comparisons between cardiac PET/MRI and PET/CT are needed especially using routine nuclear cardiology tools, such as semi-quantitative polar maps, absolute flow comparison, and regional comparisons as well. ${ }^{15,16}$ Therefore, caution speaks toward keeping routine cardiac PET on PET/CT scanners, where optimal quantification can be guaranteed and toward keeping the search for better attenuation compensation algorithms on hybrid PET/MRI systems.

\section{Disclosures}

H. Zaidi has a grant from Siemens Healthineers that is not influencing this editorial. R. Nkoulou has nothing to disclose.

\section{References}

1. Ficaro EP, Fessler JA, Shreve PD, Kritzman JN, Rose PA, Corbett JR. Simultaneous transmission/emission myocardial perfusion tomography. Diagnostic accuracy of attenuation-corrected $99 \mathrm{mTc}$ sestamibi single-photon emission computed tomography. Circulation 1996;93:463-73.

2. Martinez-Moller A, Souvatzoglou M, Delso G, Bundschuh RA, Chefd'hotel C, Ziegler SI, et al. Tissue classification as a potential approach for attenuation correction in whole-body PET/MRI: Evaluation with PET/CT data. J Nucl Med 2009;50:520-6.

3. Catana C, van der Kouwe A, Benner T, Michel CJ, Hamm M, Fenchel M, et al. Toward implementing an MRI-based PET attenuation-correction method for neurologic studies on the MRPET brain prototype. J Nucl Med 2010;51:1431-8.

4. Yang J, Wiesinger F, Kaushik S, Shanbhag D, Hope TA, Larson $\mathrm{PEZ}$, et al. Evaluation of sinus/edge-corrected zero-echo-timebased attenuation correction in brain PET/MRI. J Nucl Med 2017;58:1873-9.

5. Keereman V, Holen RV, Mollet P, Vandenberghe S. The effect of errors in segmented attenuation maps on PET quantification. Med Phys 2011;38:6010-9.

6. Rezaei A, Defrise M, Nuyts J. ML-reconstruction for TOF-PET with simultaneous estimation of the attenuation factors. IEEE Trans Med Imaging 2014;33:1563-72.

7. Mehranian A, Zaidi H. Joint estimation of activity and attenuation in whole-body TOF PET/MRI using constrained Gaussian mixture models. IEEE Trans Med Imaging 2015;34:1808-21.

8. Lassen ML, Rasul S, Beitzke D, Stelzmuller ME, Cal-Gonzalez J, Hacker M, et al. Assessment of attenuation correction for myocardial PET imaging using combined PET/MRI. J Nucl Cardiol 2017. https://doi.org/10.1007/s12350-017-1118-2.

9. Gould KL, Pan T, Loghin C, Johnson NP, Guha A, Sdringola S. Frequent diagnostic errors in cardiac PET/CT due to misregistration of CT attenuation and emission PET images: A definitive analysis of causes, consequences, and corrections. J Nucl Med 2007;48:1112-21

10. Martinez-Moller A, Souvatzoglou M, Navab N, Schwaiger M, Nekolla SG. Artifacts from misaligned CT in cardiac perfusion PET/CT studies: Frequency, effects, and potential solutions. J Nucl Med 2007;48:188-93.

11. Kustner T, Schwartz M, Martirosian P, Gatidis S, Seith F, Gilliam $\mathrm{C}$, et al. MR-based respiratory and cardiac motion correction for PET imaging. Med Image Anal 2017;42:129-44. 
12. Grimm R, Furst S, Dregely I, Forman C, Hutter JM, Ziegler SI, et al. Self-gated radial MRI for respiratory motion compensation on hybrid PET/MR systems. Med Image Comput Comput Assist Interv 2013;16:17-24.

13. Gupta M, Kadakia J, Hacioglu Y, Ahmadi N, Patel A, Choi T, et al. Non-contrast cardiac computed tomography can accurately detect chronic myocardial infarction: Validation study. J Nucl Cardiol 2011;18:96-103.

14. Fuchs TA, Ghadri JR, Stehli J, Gebhard C, Kazakauskaite E, Klaeser B, et al. Hypodense regions in unenhanced CT identify nonviable myocardium: Validation versus ${ }^{18}$ F-FDG PET. Eur J Nucl Med Mol Imaging 2012;39:1920-6.

15. Xu Y, Hayes S, Ali I, Ruddy TD, Wells RG, Berman DS, et al. Automatic and visual reproducibility of perfusion and function measures for myocardial perfusion SPECT. J Nucl Cardiol 2010;17:1050-7.

16. Valenta I, Quercioli A, Vincenti G, Nkoulou R, Dewarrat S, Rager $\mathrm{O}$, et al. Structural epicardial disease and microvascular function are determinants of an abnormal longitudinal myocardial blood flow difference in cardiovascular risk individuals as determined with PET/CT. J Nucl Cardiol 2010;17:1023-33. 